TITLE:

\title{
Preparation of gelatin hydrogels incorporating small interfering RNA for the controlled release.
}

\section{$\operatorname{AUTHOR}(\mathrm{S}):$}

Saito, Takashi; Tabata, Yasuhiko

\section{CITATION:}

Saito, Takashi ...[et al]. Preparation of gelatin hydrogels incorporating small interfering RNA for the controlled release.. Journal of drug targeting 2012, 20(10): 864-872

ISSUE DATE:

2012-12

URL:

http://hdl.handle.net/2433/184918

\section{RIGHT:}

(c) 2012 Informa Plc.; この論文は出版社版でありません。引用の際には 出版社版をご確認ご利用ください。; This is not the published version. Please cite only the published version. 
Preparation of gelatin hydrogels incorporating small interfering RNA for the controlled release

Takashi Saito and Yasuhiko Tabata *

Department of Biomaterials, Field of Tissue Engineering, Institute for Frontier Medical Sciences,

Kyoto University, 53 Kawara-cho Shogoin, Sakyo-ku Kyoto 606-8507, Japan

* Corresponding Author's Contact Information

Yasuhiko Tabata

Department of Biomaterials, Field of Tissue Engineering,

Institute for Frontier Medical Sciences, Kyoto University,

53 Kawara-cho Shogoin, Sakyo-ku Kyoto 606-8507, Japan

TEL: +81-75-751-4121

FAX: +81-75-751-4646

E-mail: yasuhiko@frontier.kyoto-u.ac.jp 


\section{ABSTRACT}

The objective of this study is to design biodegradable hydrogels of gelatin for the controlled release of bioactive small interfering RNA (siRNA). Gelatin was cationized by chemically introducing ethylene diamine into the carboxyl groups in different conditions to obtain cationized gelatins (CGs). The CG was mixed with siRNA at various mixing ratios of amino groups of gelatin to the phosphate groups of siRNA in aqueous solution to form the siRNA-CG nano-complex. Gelatin together with the complex of siRNA and CG was chemically crosslinked to prepare the gelatin hydrogel incorporating the complex. The hydrogel incorporating the siRNA-CG complex was not degraded in phosphate-buffered saline solution (PBS) at $37{ }^{\circ} \mathrm{C}$ and did not release the siRNA complex after initial siRNA release and hydrogel degradation. When placed in PBS containing collagenase, the hydrogel was enzymatically degraded to release the siRNA complex. In this release system, the siRNA was released as a result of hydrogel degradation. The siRNA released showed an activity to specifically suppress the expression of the corresponding gene. The hydrogel is promising to release siRNA and also elicit biological activity for a longer time.

Keywords: Gelatin hydrogel; Controlled release; siRNA; Gene delivery. 


\section{Introduction}

RNA interference (RNAi) is a biological phenomenon to turn off gene expression by directing degradation of the targeting mRNA, which was discovered in 1998 (Fire et al., 1998). Small interfering RNA (siRNA) is a short double-strand RNA of average only 21 - 23 base pairs which can induce RNAi (Zamore et al., 2000). Since siRNA can specifically inhibit the expression of target genes, the applications are widely being expected, for example, experimental tools to suppress gene expression in the sequence-specific manner (Gilbert et al., 2011; Tuzmen et al., 2011) and molecular-targeted therapy to inhibit the expression of pathogenic proteins related to cancer and viral infections (Gong et al., 2005; Rye et al., 2004). However, siRNA alone cannot always induce RNAi phenomena because of the low cell interaction and the low stability in the body. To effectively use siRNA-based gene suppression as both experimental and therapeutic tools, some technologies and methodologies should be established. Virus vectors have a high gene transfection efficiency and the long-term maintenance of gene expression (Xia et al., 2002), but the safety seems to be a major problem (Marshall 2002). Compared to the viral vectors, the non-viral vectors are relatively safe, however they need more enhancements for efficient introduction of genes into the target cells. Physical stimulation (e.g. electroporation (Reed et al., 2009), sonoporation (Newman et al., 2007), and iontophoresis (Andrieu-Soler et al., 2006)) enhanced the efficacy of gene transfection, although the technology is costly. To effectively use siRNA-based gene silencing as both the experimental and therapeutic tools, the level 
and duration of gene silencing need to be optimized.

Drug delivery system (DDS) is one of the practically possible technologies to positively modify the biological activity of several drugs by combination of a drug and the carrier. Gelatin is a biocompatible protein which can be obtained through an alkaline, acid or enzymatic processing of animals' collagen. Gelatin has various side chains (e.g. amino and carboxyl groups) which can be chemically modified with ease. Dehydrothermal or chemical treatment enables gelatin to intermolecularly crosslink for the hydrogel formation. Gelatin hydrogel is generally degraded by enzymes, such as collagenase and gelatinase of matrix metalloproteinases (MMPs) (Egeblad et al., 2002) and the hydrogel degradability can be changed by altering the crosslinking conditions. We have designed various hydrogels of DDS carriers with gelatin and the chemical derivatives to release biologically active substances, such as basic fibroblast growth factor (Tabata et al., 2000), bone morphogenetic protein 2 (Kimura et al., 2010), and transforming growth factor - $\beta 1$ (Holland et al., 2004), plasmid DNA (Kushibiki et al., ; Lin et al., 2009), siRNA (Matsumoto et al., 2006), or low molecular compounds (Tanigo et al., 2010).

The objective of this study is to create a gelatin hydrogel system for the controlled release of bioactive siRNA. Cationized gelatin (CG) was prepared to form the polyion complex of siRNA. The complex was mixed with gelatin, followed by chemical crosslinking to prepare gelatin hydrogels incorporating the complex of siRNA and CG. The siRNA release profile from the hydrogel was investigated while the activity of siRNA released was evaluated. 


\section{Materials and Methods}

\subsection{Materials}

Gelatin with an isoelectric point $(\mathrm{pI})$ of $5.0(\mathrm{Mw}=100,000)$, prepared through an alkaline process of bovine bone (pI5 gelatin) or with a $\mathrm{pI}$ of $9.0(\mathrm{Mw}=100,000)$, prepared through an acid process of pig skin (pI9 gelatin) and collagenase L were kindly supplied from Nitta Gelatin Co., Osaka, Japan. The nucleotide sequences of siRNA targeted at the firefly luciferase gene (Luc siRNA) (Forward) 5'CUUACGCUGAGUACUUCGATT -3' and (Reverse) 5'- UCGAAGUACUCAGCGUAAGTT -3' was purchased from B-Bridge International (Sunnyvale, CA, USA). All reagents were obtained from Nacalai Tesque, Kyoto, Japan except as indicated otherwise.

\subsection{Preparation of Luc siRNA and cationized gelatin complexes}

The cationized gelatins (CGs) with different cationization extents were prepared as described previously (Saito et al., 2012). Briefly, various amounts of ethylenediamine (EDA) were added into $25 \mathrm{ml}$ of $0.1 \mathrm{M}$ phosphate-buffered solution $(\mathrm{PB}, \mathrm{pH}=5.0)$ containing $1.0 \mathrm{~g}$ of pI9 gelatin. The solution $\mathrm{pH}$ was adjusted to 5.0 by adding $6 \mathrm{M} \mathrm{HCl}$ aqueous solution, and $\mathrm{PB}$ was added into the solution to give the final volume of $50 \mathrm{ml}$. Then, 1-ethyl-3-(3-dimethylaminopropyl) carbodiimide hydrochloride (EDC) was added into the solution, followed by the agitation at $37{ }^{\circ} \mathrm{C}$ for $4 \mathrm{hr}$ and dialysis against double-distilled water (DDW) for 3 days at room temperature. The dialyzed solution 
was freeze-dried to obtain CGs. Table 1 summarizes the molar percentage of EDA introduced to the carboxyl groups of gelatin. Next, the complexation of Luc siRNA and CG was performed by simply mixing at various mixing ratios of amino group number of CG per the phosphate group number of Luc siRNA (N/P) in aqueous solution. Briefly, deionized and sterilized water (RNase-free, DSW) containing different amounts of CG was slowly added to the same volume of $100 \mathrm{mM}$ phosphate-buffered saline solution (PBS, $\mathrm{pH}=7.4)$ containing $10 \mathrm{nM}$ of Luc siRNA at N/P mixing ratios of $1,5,10,20,30$, and 50 . The mixed solution was gently agitated at $37{ }^{\circ} \mathrm{C}$ for $10 \mathrm{~min}$ to form Luc siRNA and CG complexes. Table 1 summarizes the amount of CG against $1 \mu \mathrm{M}$ of Luc siRNA at N/P ratio 10. The CGs were sterilized by ethylene oxide gas (EOG) on a EOGelk SA-360ECO (ELK corporation, Osaka, Japan) before using.

2.3. Dynamic light scattering and zeta potential measurements of CG complexes with Luc SiRNA

To evaluate the nanocomplex size and zeta potential of CG and the Luc siRNA complexes, dynamic light scattering (DLS) and electrophoretic light scattering (ELS) measurements were carried out as described previously (Saito et al., 2012). The DLS measurement was carried out on aDLS-DPA-60HD (Otsuka Electronic Co.Ltd., Osaka, Japan) equipped with a $\mathrm{He}-\mathrm{Ne}$ laser at a detection angle of $90^{\circ}$ at $37^{\circ} \mathrm{C}$. ELS (ELS-7000, Otsuka Electronic Co. Ltd., Osaka, Japan) was 
measured at room temperature and an electric field strength of $100 \mathrm{~V} / \mathrm{cm}$. The experiment was done

three times independently for every sample unless otherwise mentioned.

\subsection{Cytotoxicity evaluation of CG}

A luciferase-expressing cell line derived from colon 26 (Colon26-Luc) was kindly supplied by Dr.

Takakura of Graduate School of Pharmaceutical Sciences, Kyoto University, Kyoto, Japan. The

Colon26-Luc cells were cultivated in the Dulbecco's modified Eagle medium (DMEM) containing 10 vol\% fetal bovine serum (FBS) and 1.0 vol\% penicillin and streptomycin (DMEM-FBS) at $37{ }^{\circ} \mathrm{C}$ in a $95 \%$ air $-5 \% \mathrm{CO} 2$ atmosphere. The cytotoxicity of CG was evaluated by an in vitro bioassay as described previously (Saito et al., 2012) with minor modification. Briefly, Colon26-Luc cells were seeded into each well of 96-well multi-well culture plate (Corning Inc., NY, USA) at a density of 5,000 cells $(100 \mu \mathrm{l})$ per well for $24 \mathrm{hr}$. And then, the medium was exchanged to $90 \mu \mathrm{l}$ of fresh medium and $10 \mu \mathrm{l}$ of $\mathrm{CG}$ aqueous solution, and $24 \mathrm{hr}$ later, 2-(2-methoxy-4-nitrophenyl)-3-(4-nitrophenyl)-5-(2,4-disulfophenyl)-2H-tetrazolium (WST-8) of cell count reagent SF (Nacalai Tesque, Kyoto, Japan) $(10 \mu \mathrm{l})$ was added into each well followed by incubation further for $30 \mathrm{~min}$. The absorbance of medium was measured at $450 \mathrm{~nm}$ by the VERSAmax microplate reader (Molecular Devices, Sunnyvale CA, USA). The percentage of cell viability was expressed as $100 \%$ for cells cultured without gelatin. The experiment was done 5 times independently for each sample. 


\subsection{Evaluation of gene suppression of Luc siRNA and CG complexes}

Gene suppression with Luc siRNA and CG complexes were evaluated according to the method reported previously (Nagane et al., 2009). Colon26-Luc cells were seeded into each well of 12-well multi-well culture plate (Corning Inc., NY, USA) at a density of 50,000 cells (1.0 ml) per well in the DMEM. After $24 \mathrm{hr}$ incubation, the medium was removed, and the well was washed by PBS $(1.0 \mathrm{ml})$ twice. Then, Opti-MEM I Reduced-Serum Medium (Opti-MEM, Life Technologies Japan Ltd., Osaka, Japan) of gene transfection medium $(450 \mu \mathrm{l})$ and the complex solution of Luc siRNA at different concentrations of CG $(50 \mu \mathrm{l})$ were added into each well, followed by $6 \mathrm{hr}$ incubation. After rinsing with PBS, cells were continued to culture in DMEM-FBS further for 0, 18, and $42 \mathrm{hr}$. To evaluate the biological activity of Luc siRNA, cells were washed with PBS twice, lysed in $100 \mu$ of cell-culture lysis reagent (Promega KK, Osaka, Japan). Then, $100 \mu$ l luciferase assay reagent (Promega KK, Osaka, Japan) was added to $10 \mu \mathrm{l}$ of supernatant while the relative light unit (RLU) of the samples was determined by a luminometer (MicroLumatPlus LB 96V, Berthold, Tokyo, Japan). The total protein content in each well was determined by micro bicinchonic acid (BCA) protein assay kit (Pierce, Rockford, IL, USA) according to the manufacturers' instructions to normalize the influence of number variance of cells on the luciferase activity. Each experimental group was carried out three times independently. 


\subsection{Preparation of gelatin hydrogels incorporating Luc siRNA and CG complexes}

To prepare the complex of Luc siRNA and CG, Luc siRNA (10 nmole/ml, $100 \mu \mathrm{l})$ was mixed with various N/P ratios of CG in DSW (100 $\mu \mathrm{l})$ at $37{ }^{\circ} \mathrm{C}$. Next, aqueous solution of pI5 gelatin $(20 \mathrm{wt} \%$, $200 \mu \mathrm{l}$ ) was added to the complex solution at $37{ }^{\circ} \mathrm{C}$. Then, the solution was mixed with $25 \mathrm{wt} \%$ of glutaraldehyde $(2.5 \mu \mathrm{l})$ at a concentration of $0.63 \mathrm{vol} \%$, and cast into a polypropylene dish (1.0 $\mathrm{mm} \times 1.0 \mathrm{~mm}$, Sakura Finetek Japan Co., Ltd., Tokyo, Japan), followed by leaving at $4{ }^{\circ} \mathrm{C}$ for $8 \mathrm{hr}$ for chemical crosslinking of gelatin. The hydrogels were agitated in $100 \mathrm{mM}$ glycine solution at $37{ }^{\circ} \mathrm{C}$ for $1 \mathrm{hr}$ to block the residual aldehyde groups of glutaraldehyde. Following $1 \mathrm{hr}$ washing three times with DSW, the hydrogels were freeze-dried to obtain the gelatin hydrogels incorporating Luc siRNA and CG complex.

2.7. Release test of Luc siRNA from gelatin hydrogels incorporating Luc siRNA and CG complexes

Hydrogels of 5.0 to $7.0 \mathrm{mg}$ in dry weight were placed in PBS $(500 \mu \mathrm{l})$ at $37{ }^{\circ} \mathrm{C}$. The PBS supernatant was exchanged $1,2,4,8,24,48$, and $96 \mathrm{hr}$ later and subsequently the hydrogels were completely degraded by PBS containing collagenase $(5.0 \mu \mathrm{g} / \mathrm{ml}, 500 \mu \mathrm{l})$. The release test was performed in PBS for the initial $24 \mathrm{hr}$ and after that done in PBS containing collagenase $(5.0 \mu \mathrm{g} / \mathrm{ml})$. The amount of Luc siRNA in solution sampled was determined by the conventional method of 
ethidium bromide (Et-Br) with fluorescence spectrometry (Le Pecq et al., 1966). Briefly, Et-Br was dissolved in $0.1 \mathrm{M} \mathrm{NaCl}$ and $0.1 \mathrm{M}$ tris (hydroxymethyl) aminomethane aqueous solution $(10 \mu \mathrm{g} / \mathrm{ml})$. The Et-Br solution $(150 \mu \mathrm{l})$ and sample solution $(150 \mu \mathrm{l})$ were mixed in each well of 96 -well multi-well culture black plate (Corning Inc., NY, USA) A calibration curve was prepared with the determined amounts of Luc siRNA. Fluorescence spectra were measured at room temperature on a SpectraMax Gemini EM (Molecular Device, Osaka, Japan) at the excitation and emission wavelengths of 546 and $596 \mathrm{~nm}$, respectively. The percent introduced was determined based on the calibration curve and the fluorescence of samples. The experiment was done three times independently for every sample unless otherwise mentioned.

\subsection{Degradation evaluation of hydrogels incorporating Luc siRNA and CG complex}

Hydrogels of 5.0 to $7.0 \mathrm{mg}$ in dry weight were placed in PBS $(500 \mu \mathrm{l})$ at $37{ }^{\circ} \mathrm{C}$. The PBS supernatant was exchanged 1, 2, 4, 8, and $24 \mathrm{hr}$ later and subsequently PBS containing collagenase $(5.0 \mu \mathrm{g} / \mathrm{ml})$ was added. PBS containing collagenase supernatant was exchanged every $3 \mathrm{hr}$ and fresh one was added. The amount of gelatin solution sampled was determined by micro BCA protein assay described above. 


\subsection{Evaluation of gene suppression experiments of Luc siRNA released from hydrogels}

The hydrogel of 5.0 to $7.0 \mathrm{mg}$ in dry weight was placed in Opti-MEM $(1.0 \mathrm{ml})$ at $37{ }^{\circ} \mathrm{C}$. The Opti-MEM supernatant was collected $24 \mathrm{hr}$ later, and then $1.0 \mathrm{ml}$ of Opti-MEM containing collagenase (5.0 $\mu \mathrm{g} / \mathrm{ml}$, Opti-MEM-col) was added. The Opti-MEM-col was exchanged 3, 6, 9, and $12 \mathrm{hr}$ later to collect the supernatant. The hydrogel was gradually degraded in Opti-MEM-col with time to release the Luc siRNA incorporated. The amount of gelatin in the supernatant sampled was determined by the micro BCA protein assay described above. The Luc siRNA concentration of supernatant sampled was determined by the Et-Br fluorescence spectrometry described above. The gene suppression activity of Luc siRNA in the supernatants collected, was performed according to the method previously reported (Nagane et al., 2009). Briefly, Colon26-Luc cells were cultured in each well of 12-well multi-well culture plate at a density of 50,000 cells $(1.0 \mathrm{ml})$ in the DMEM for $24 \mathrm{hr}$ at $37^{\circ} \mathrm{C}$ in $5 \% \mathrm{CO}-95 \%$ air atmosphere. After washing of the well by PBS (1.0 ml) twice, the supernatant of Opti-MEM or Opti-MEM-col $(500 \mu \mathrm{l})$ was added into each well, followed by $6 \mathrm{hr}$ incubation. The luciferase and micro BCA protein assays were performed described above. As a control, the similar experiment for the complex of Luc siRNA freshly prepared at the same concentration as the supernatant collected at the corresponding N/P ratio and CG type was carried out. The percent suppression activity was calculated using the following formula. 
Suppression activity $(\%)=(100-$ percent luciferase expression of supernatant $) \times 100 /(100-$ percent luciferase expression of control complex)

The experiment was done three times independently for every sample unless otherwise mentioned.

\subsection{Statistical analysis}

All the results were expressed as the mean \pm standard deviation (SD). Significant analysis was done based on Fisher's least significant difference test for multiple comparisons, and the difference was considered to be significant at $\mathrm{p}<0.05$. 


\section{RESULTS}

\subsection{Characterization of Luc siRNA and CG complexes}

Table 1 summarizes the preparation of CG and the complexes with Luc siRNA. Figure 1 shows the apparent molecular size and zeta potential of CG with different cationization extents and the Luc siRNA complexes. The molecular size of CGs and the Luc siRNA complex tended to reach to a certain value when the N/P ratio was higher than 10 . The complexes size ranged from 100 to $300 \mathrm{~nm}$ at higher N/P ratios. The zeta potential of CGs and the Luc siRNA complexes tended to increase with the cationization extent of gelatin and N/P mixing ratio.

Figure 2 shows the cytotoxicity of CGs. CGs with lower extents of cationization showed no cytotoxicity. On the contrary, the E-7.0 and E-50 CGs showed cytotoxicity. Overall, the cytotoxicity increased with an increase in the CG amount.

\subsection{Gene suppression of Luc siRNA and CG complexes}

Figure 3 shows the gene expression suppression of Luc siRNA and CG complexes. Higher suppression of gene expression was observed for the Luc siRNA complexes of E-7.0 and E-50 CGs than those of E-1.0 and E-3.0 ones. The E-50 complex showed the strongest and longest gene suppression. 
3.3. In vitro time profiles of Luc siRNA release from gelatin hydrogels incorporating Luc siRNA and CG complexes

Figure 4 shows the in vitro time profiles of Luc siRNA release from gelatin hydrogels incorporating Luc siRNA and CG complexes in PBS with or without collagenase. About $35 \%$ of Luc siRNA was released initially, but after that no release was observed. On the other hand, in PBS containing collagenase, the Luc siRNA was released similarly, irrespective of the CG type and N/P ratio.

\subsection{In vitro time profiles of hydrogel degradation}

Figure 5 shows in vitro time profiles of gelatin hydrogel degradation in PBS with or without collagenase. About $29 \%$ of hydrogel was degraded initially, but after that no degradation was observed. On the other hand, in PBS containing collagenase, the hydrogel was degraded similarly, irrespective of the CG type and N/P ratio.

\subsection{Gene suppression activity of Luc siRNA released}

Figure 6 shows the gene suppression activity of medium supernatants after culture with hydrogels incorporating Luc siRNA and CG complex. The Opti-MEM supernatant did not show the suppression activity of gene expression (Figure 6 (b)) irrespective of the N/P ratio and CG type. On 
the contrary, the Opti-MEM-col supernatant had significant suppression activity (Figure 6 (a)). The suppression activity for gelatin hydrogels incorporating Luc siRNA and CG complexes at the N/P ratio of 50 had significantly higher than that of hydrogels at the N/P ratio of 10 (Figure 6 (c). Even after 3, 6, and $9 \mathrm{hr}$ incubation in Opti-MEM-col, the supernatants showed significant gene suppression activity (Figure 6 (d)). However, after $12 \mathrm{hr}$ incubation in Opti-MEM-col, any supernatant did not show gene suppression activity because the amount of Luc siRNA in the supernatant was very low (data not shown). Table 3 summarizes the amount of Luc siRNA released from gelatin hydrogels in Opti-MEM-col. Luc siRNA was gradually released from gelatin hydrogel and the released amount of siRNA maintained the suppression activity (Figure $6(d)$ ). 


\section{Discussion}

The present study demonstrates that the biological activity of siRNA was associated with the release rate of siRNA from the hydrogel and to the enzymatic degradation of gelatin hydrogels.

Gelatin and the derivatives have been used to prepare the hydrogel of carrier material for the controlled release of various drugs (Tanigo et al., 2010), proteins (Kohara et al., 2011; Ratanavaraporn et al., 2011), and genes (Doi et al., 2011; Kushibiki et al., 2004; Lin et al., 2009; Matsumoto et al., 2006), because of the biodegradability and biocompatibility. When the siRNA was directly incorporated into the hydrogels prepared by the chemical crosslinking of pI5 or pI9 gelatin, an initial burst in siRNA release (about $80 \%$ ) was observed (data not shown). For hydrogels prepared from pI5 gelatin (5 wt\%), an initial burst in siRNA release (34.3 to $53.4 \%$ ) was observed (Supplementary Figure 1). This is because the siRNA molecules do not always interact with the gelatin molecules, resulting in the burst release by the simple diffusion of siRNA. One of the methods to enhance the interaction of siRNA with gelatin is to use the cationized derivative which can electrostatically interact with negatively charged siRNA. The hydrogel of cationized gelatin worked well to achieve the controlled release of siRNA in vivo (Obata et al., 2012). However, the cationized hydrogel sometimes induces severe inflammation reaction compared to the normal gelatin. Therefore, the siRNA of negative charge (phosphate group) was mixed with the 
$\mathrm{CG}$ to form the nano-complex. Then, the complex was mixed with aqueous gelatin solution at a high concentration $(20 \mathrm{wt} \%)$ to obtain the hydrogels. The complex of positive charge enabled the immobilization into the hydrogel of pI5 gelatin with a negative charge. In this release system, the siRNA is released in the complex form with the CG hydrogel only when the hydrogel is enzymatically degraded to generate water-soluble gelatin fragments. The siRNA activity to suppress the gene expression is high for the complex form compared with siRNA in the free form (Kim et al., 2010). It is possible that the siRNA was released from the hydrogel in the form of complex with CG. CG will accelerate the cellular internalization of siRNA, resulting in the enhanced gene suppression activity.

Several researchers investigate the carrier for the sustained release of gene in vitro (Jensen et al., 2012; Krebs et al., 2009; Nelson et al., 2012) and in vivo (Jacobson et al., 2010; Lobovkina et al., 2011). For example, the hydrogels of photo-crosslinked alginate and collagen achieved the sustained delivery of siRNA (Krebs et al., 2009). The 3-dimensional hydrogels for sustained delivery of siRNA could suppress gene expression for 6 days in vitro. In addition, the in vivo sustained release of siRNA could be achieved with solid lipid nanoparticles (Lobovkina et al., 2011). The present hydrogel system is applicable for the sustained release of siRNA as a result of enzymatic hydrogel degradation. The release profiles of siRNA were in consistence with the rate of hydrogel degradation. This again supports the release of siRNA in the complex form with the degraded fragment of CG. In 
the case of in vitro and in vivo controlled siRNA release study, the hydrogel system can be changed the release profile by using different concentration of collagenase solutions or different crosslinking extent of the hydrogels. The time period of hydrogels degradation ranged from a few days to several months (Tabata et al., 1999).These controlled gene silencing technologies have significant potential for use in tissue regeneration applications and experimental tools for suppression of specific gene expression.

The key factor to consider the siRNA action is not only the extent of gene silencing, but also the time period of activity maintenance. Even if the siRNA can be released, the release of inactive siRNA is practically useless. Therefore, in this study, the gene suppression activity of supernatants after the placement of hydrogel in the Opti-MEM-col was assessed (Figures 6 (a-d)). When placed in Opti-MEM-col, it is likely that the hydrogel was degraded to release the Luc siRNA complex incorporated in the hydrogel. When compared at the same siRNA concentration, the supernatant containing the complex released exhibited significant gene suppression activity although the activity was low compared with the fresh complex as a control. siRNA released from gelatin hydrogels incorporating Luc siRNA and CG complex (N/P ratio 50) showed higher transfection efficacy than those of $\mathrm{CG}$ at the N/P ratio of 10 . It is highly conceivable that during enzymatic degradation, the hydrogel gelatin and CG for the complexation with siRNA were randomly degraded. However, the siRNA activity in the supernatant after hydrogel incubation in the Opti-MEM-col remained. It is 
highly conceivable that even by enzymatic degradation, the siRNA itself was not degraded because of the complexation. The siRNA would be released in the complex form. In conclusion, the biodegradable hydrogel of gelatin is a promising carrier for the siRNA release which is driven by the hydrogel degradation. The Opti-MEM-col supernatants showed an activity to suppress the expression of the corresponding gene. By using the hydrogel, the biological activity of siRNA can be retained for a long time period.

\section{References}

C. Andrieu-Soler, M. Doat, M. Halhal, N. Keller, L. Jonet, D. BenEzra, F. Behar-Cohen, (2006). Enhanced oligonucleotide delivery to mouse retinal cells using iontophoresis. Molecular vision, 12, 1098-1107.

N. Doi, J.I. Jo, Y. Tabata, (2011). Preparation of Biodegradable Gelatin Nanospheres with a Narrow Size Distribution for Carrier of Cellular Internalization of Plasmid DNA. J Biomater Sci Polym Ed, 23, 991-1004.

M. Egeblad, Z. Werb, (2002). New functions for the matrix metalloproteinases in cancer progression. Nat Rev Cancer, 2, 161-174.

A. Fire, S. Xu, M.K. Montgomery, S.A. Kostas, S.E. Driver, C.C. Mello, (1998). Potent and specific genetic interference by double-stranded RNA in Caenorhabditis elegans. Nature, 391, 806-811.

D.F. Gilbert, G. Erdmann, X. Zhang, A. Fritzsche, K. Demir, A. Jaedicke, K. Muehlenberg, E.E. Wanker, 
M. Boutros, (2011). A novel multiplex cell viability assay for high-throughput RNAi screening. PloS one,

6, e28338.

H. Gong, C.M. Liu, D.P. Liu, C.C. Liang, (2005). The role of small RNAs in human diseases: potential troublemaker and therapeutic tools. Medicinal research reviews, 25, 361-381.

T.A. Holland, J.K. Tessmar, Y. Tabata, A.G. Mikos, (2004). Transforming growth factor-beta 1 release from oligo(poly(ethylene glycol) fumarate) hydrogels in conditions that model the cartilage wound healing environment. J Control Release, 94, 101-114.

G.B. Jacobson, E. Gonzalez-Gonzalez, R. Spitler, R. Shinde, D. Leake, R.L. Kaspar, C.H. Contag, R.N. Zare, (2010). Biodegradable nanoparticles with sustained release of functional siRNA in skin. Journal of pharmaceutical sciences, 99, 4261-4266.

D.K. Jensen, L.B. Jensen, S. Koocheki, L. Bengtson, D. Cun, H.M. Nielsen, C. Foged, (2012). Design of an inhalable dry powder formulation of DOTAP-modified PLGA nanoparticles loaded with siRNA. J Control Release, 157, 141-148.

H.J. Kim, A. Ishii, K. Miyata, Y. Lee, S. Wu, M. Oba, N. Nishiyama, K. Kataoka, (2010). Introduction of stearoyl moieties into a biocompatible cationic polyaspartamide derivative, PAsp(DET), with endosomal escaping function for enhanced siRNA-mediated gene knockdown. J Control Release, 145, 141-148.

Y. Kimura, N. Miyazaki, N. Hayashi, S. Otsuru, K. Tamai, Y. Kaneda, Y. Tabata, (2010). Controlled release of bone morphogenetic protein-2 enhances recruitment of osteogenic progenitor cells for de novo 
generation of bone tissue. Tissue Eng Part A, 16, 1263-1270.

H. Kohara, Y. Tabata, (2011). Enhancement of ectopic osteoid formation following the dual release of bone morphogenetic protein 2 and Wnt1 inducible signaling pathway protein 1 from gelatin sponges.

Biomaterials, 32, 5726-5732.

M.D. Krebs, O. Jeon, E. Alsberg, (2009). Localized and sustained delivery of silencing RNA from macroscopic biopolymer hydrogels. Journal of the American Chemical Society, 131, 9204-9206.

T. Kushibiki, K. Matsumoto, T. Nakamura, Y. Tabata, (2004). Suppression of the progress of disseminated pancreatic cancer cells by NK4 plasmid DNA released from cationized gelatin microspheres. Pharm Res, 21, 1109-1118.

J.B. Le Pecq, C. Paoletti, (1966). A new fluorometric method for RNA and DNA determination. Anal Biochem, 17, 100-107.

X. Lin, H. Jo, T.M. Ishii, M. Fujita, M. Fu, K. Tambara, M. Yamamoto, Y. Tabata, M. Komeda, S. Matsuoka, (2009). Controlled release of matrix metalloproteinase-1 plasmid DNA prevents left ventricular remodeling in chronic myocardial infarction of rats. Circ J, 73, 2315-2321.

T. Lobovkina, G.B. Jacobson, E. Gonzalez-Gonzalez, R.P. Hickerson, D. Leake, R.L. Kaspar, C.H. Contag, R.N. Zare, (2011). In vivo sustained release of siRNA from solid lipid nanoparticles. ACS nano, 5, 9977-9983.

E. Marshall, (2002). Gene therapy. What to do when clear success comes with an unclear risk? Science, 
$298,510-511$.

G. Matsumoto, T. Kushibiki, Y. Kinoshita, U. Lee, Y. Omi, E. Kubota, Y. Tabata, (2006). Cationized gelatin delivery of a plasmid DNA expressing small interference RNA for VEGF inhibits murine squamous cell carcinoma. Cancer Sci, 97, 313-321.

K. Nagane, M. Kitada, S. Wakao, M. Dezawa, Y. Tabata, (2009). Practical induction system for dopamine-producing cells from bone marrow stromal cells using spermine-pullulan-mediated reverse transfection method. Tissue Eng Part A, 15, 1655-1665.

C.E. Nelson, M.K. Gupta, E.J. Adolph, J.M. Shannon, S.A. Guelcher, C.L. Duvall, (2012). Sustained local delivery of siRNA from an injectable scaffold. Biomaterials, 33, 1154-1161.

C.M. Newman, T. Bettinger, (2007). Gene therapy progress and prospects: ultrasound for gene transfer. Gene therapy, 14, 465-475.

Y. Obata, T. Nishino, T. Kushibiki, R. Tomoshige, Z. Xia, M. Miyazaki, K. Abe, T. Koji, Y. Tabata, S. Kohno, (2012). HSP47 siRNA conjugated with cationized gelatin microspheres suppresses peritoneal fibrosis in mice. Acta biomaterialia, 8, 2688-2696.

J. Ratanavaraporn, H. Furuya, H. Kohara, Y. Tabata, (2011). Synergistic effects of the dual release of stromal cell-derived factor-1 and bone morphogenetic protein-2 from hydrogels on bone regeneration.

Biomaterials, 32, 2797-2811.

S.D. Reed, S. Li, (2009). Electroporation advances in large animals. Curr Gene Ther, 9, 316-326. 
P.D. Rye, T. Stigbrand, (2004). Interfering with cancer: a brief outline of advances in RNA interference in oncology. Tumour biology : the journal of the International Society for Oncodevelopmental Biology and Medicine, 25, 329-336.

T. Saito, Y. Tabata, (2012). Preparation of gelatin hydrogels incorporating low-molecular-weight heparin for anti-fibrotic therapy. Acta biomaterialia, 8, 646-652.

Y. Tabata, A. Nagano, Y. Ikada, (1999). Biodegradation of hydrogel carrier incorporating fibroblast growth factor. Tissue Eng, 5, 127-138.

Y. Tabata, M. Miyao, T. Inamoto, T. Ishii, Y. Hirano, Y. Yamaoki, Y. Ikada, (2000). De novo formation of adipose tissue by controlled release of basic fibroblast growth factor. Tissue Eng, 6, 279-289.

T. Tanigo, R. Takaoka, Y. Tabata, (2010). Sustained release of water-insoluble simvastatin from biodegradable hydrogel augments bone regeneration. J Control Release, 143, 201-206.

S. Tuzmen, P. Tuzmen, S. Arora, S. Mousses, D. Azorsa, (2011). RNAi-based functional pharmacogenomics. Methods Mol Biol, 700, 271-290.

H. Xia, Q. Mao, H.L. Paulson, B.L. Davidson, (2002). siRNA-mediated gene silencing in vitro and in vivo. Nat Biotechnol, 20, 1006-1010.

P.D. Zamore, T. Tuschl, P.A. Sharp, D.P. Bartel, (2000). RNAi: double-stranded RNA directs the ATP-dependent cleavage of mRNA at 21 to 23 nucleotide intervals. Cell, 101, 25-33. 
Table 1. Preparation of CG for complexation with Luc siRNA

\begin{tabular}{llllll}
\hline Code & $\begin{array}{l}\text { Gelatin concentration } \\
(\mathrm{g} / \mathrm{ml})\end{array}$ & $\begin{array}{l}\text { EDA concentration } \\
(\mathrm{ml})\end{array}$ & $\begin{array}{l}\text { EDA used } \\
\text { molar ratio }\end{array}$ & $\begin{array}{l}\text { Percentage } \\
\text { of EDA introduced }^{\mathrm{b}}\end{array}$ & $\begin{array}{l}\text { Amount of CG } \\
\text { used for complexation }^{\mathrm{c}}(\mathrm{mg} / \mathrm{ml})\end{array}$ \\
\hline E-1.0 & 1.0 & 0.063 & 1.0 & $16.7 \pm 1.8$ & 9.87 \\
E-3.0 & 1.0 & 0.188 & 3.0 & $27.0 \pm 2.8$ & 8.35 \\
E-7.0 & 1.0 & 0.439 & 7.0 & $36.0 \pm 1.2$ & 7.37 \\
E-50 & 1.0 & 3.14 & 50 & $56.5 \pm 4.5$ & 5.80 \\
\hline
\end{tabular}

${ }^{\mathrm{a}}$ The molar ratio of EDA added to the carboxyl groups of gelatin

${ }^{b}$ The molar percentage of EDA introduced to the carboxyl groups of gelatin

${ }^{\mathrm{c}}$ The amount of CG for $1 \mu \mathrm{M}$ of Luc siRNA at the N/P ratio 10 
Table 2. The amount of Luc siRNA released in Opti-MEM with or without collagenase

\begin{tabular}{lll}
\hline Code & \multicolumn{2}{c}{ Luc siRNA released in Opti-MEM (nM) } \\
\cline { 2 - 3 } & without collagenase & with collagenase \\
\hline E-7.0, N/P = 10 & $85.3 \pm 10.5$ & $126.9 \pm 16.7$ \\
E-7.0, N+P = 50 & $75.0 \pm 5.7$ & $120.0 \pm 24.5$ \\
E-50, N/P = 10 & $76.3 \pm 0.72$ & $117.5 \pm 43.8$ \\
E-50, N/P = 50 & $80.6 \pm 15.2$ & $132.6 \pm 4.7$ \\
\hline
\end{tabular}


Table 3. The amount of Luc siRNA released in Opti-MEM contains collagenase

\begin{tabular}{rlllll}
\hline \multirow{2}{*}{ Time (hr) } & \multicolumn{2}{c}{$\mathrm{E}-7.0(\mathrm{nM})$} & & \multicolumn{2}{c}{$\mathrm{E}-50(\mathrm{nM})$} \\
\cline { 2 - 3 } \cline { 5 - 6 } \cline { 5 - 6 } & $\mathrm{N} / \mathrm{P}=10$ & $\mathrm{~N} / \mathrm{P}=50$ & & $\mathrm{~N} / \mathrm{P}=10$ & $\mathrm{~N} / \mathrm{P}=50$ \\
\hline 3 & $27.3 \pm 2.3$ & $27.7 \pm 6.7$ & & $37.9 \pm 1.5$ & $22.4 \pm 3.8$ \\
6 & $32.0 \pm 3.3$ & $32.4 \pm 6.3$ & & $35.5 \pm 4.7$ & $27.1 \pm 7.6$ \\
9 & $13.2 \pm 2.9$ & $6.2 \pm 4.5$ & & $11.6 \pm 7.7$ & $15.9 \pm 6.3$ \\
12 & $3.7 \pm 2.2$ & $1.6 \pm 1.2$ & & $3.6 \pm 1.4$ & $12.9 \pm 7.5$ \\
\hline
\end{tabular}


Figure 1. Apparent molecular sizes (A) and zeta potentials (B) of Luc siRNA and CG complexes. The complexes were prepared for $1.0(\bigcirc), 10,(\triangle)$, and $100 \mathrm{nM}(\square)$ of Luc siRNA with E-1.0 (a), E-3.0 (b), E-7.0 (c), and E-50 CGs (d) in DDW at $37^{\circ} \mathrm{C}$.

Figure 2. Cytotoxicity of CGs with different cationization extents. Colon26-Luc cells were cultured in the fresh medium containing CG at concentrations of $0.1(\square), 1.0(\square)$, and $10 \mathrm{nM}(\square)$ for $24 \mathrm{hr}$. The percentage of cell viability was expressed as $100 \%$ for cells cultured in fresh medium containing pI9 gelatin. *, $\mathrm{P}<0.05$; significant against the viability of cells cultured in the medium containing pI9 gelatin.

Figure 3. Gene suppression of Luc siRNA and CG complexes. The complexes were prepared for 100 nM of Luc siRNA with E-1.0 (a), E-3.0 (b), E-7.0 (c), and E-50 CGs (d) in DSW at the N/P ratio of 1

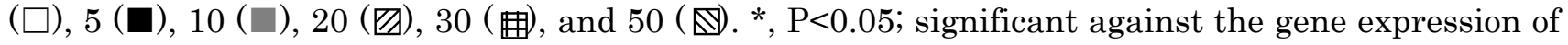
colon26-Luc without Luc siRNA.

Figure 4. In vitro release profiles of Luc siRNA from gelatin hydrogels incorporating complexes of Luc siRNA and CG without collagenase ( $\mathrm{a}$ and b). The complexes were prepared for siRNA with E-1.0 (○), E-3.0 (○), E-7.0 ( $\square$ ), and E-50 CGs ( $\boldsymbol{\square})$. The N/P ratios are 10 (a) and 50 (b). (c) In vitro release profiles of Luc siRNA from gelatin hydrogels incorporating complexes of Luc siRNA and E-7.0 CG at N/P ratios of $10(\mathrm{O})$ and $50(\mathbf{O})$ and E-50 CG, at N/P ratios of $10(\square)$ and $50(\boldsymbol{\square})$. The release test was performed in PBS for the initial $24 \mathrm{hr}$, and thereafter in 
collagenase PBS solution $(5 \mu \mathrm{g} / \mathrm{ml})$ indicated by $\mathrm{hr}$ arrow at $37^{\circ} \mathrm{C}$.

Figure 5. In vitro degradation profiles of gelatin hydrogels incorporating complex of siRNA and E-7.0, at N/P ratios of $10(\mathrm{O})$ and $50(\mathbf{O})$ and E-50 CG, at N/P ratios of $10(\square)$ and $50(\mathbf{\square})$. The degradation test was performed in PBS for the initial $24 \mathrm{hr}$, and thereafter in collagenase PBS solution $(5 \mu \mathrm{g} / \mathrm{ml})$ indicated by hr arrow at $37{ }^{\circ} \mathrm{C}$.

Figure 6. Percent gene expression of Luc siRNA released from gelatin hydrogels incorporating Luc siRNA and CG complexes in Opti-MEM with (a) and without collagenase (b). Gene suppression of fresh Luc siRNA and CG complex ( $\square$ ) and the supernatant ( $\mathbf{\square}) 24 \mathrm{hr}$ after incubation with hydrogels incorporating complex in Opti-MEM. *, $\mathrm{P}<0.05$; significant between the two groups. (c) Percent suppression activity calculated by the formulation described above. *, $\mathrm{P}<0.05$; significant between the two groups. (d) Percent gene expression of Luc siRNA released from gelatin hydrogels incorporating Luc siRNA and CG complexes in Opti-MEM with collagenase. The supernatant was collected $3(\square), 6(\square)$, and $9 \mathrm{hr}(\square)$ after culturing with the hydrogels incorporating complex to check the gene suppression activity. *, P $<0.05$; significant against the gene expression of colon26-Luc without Luc siRNA. The percentage of luciferase expression was expressed as $100 \%$ for colon26-Luc cultured in Opti-MEM without Luc siRNA. 

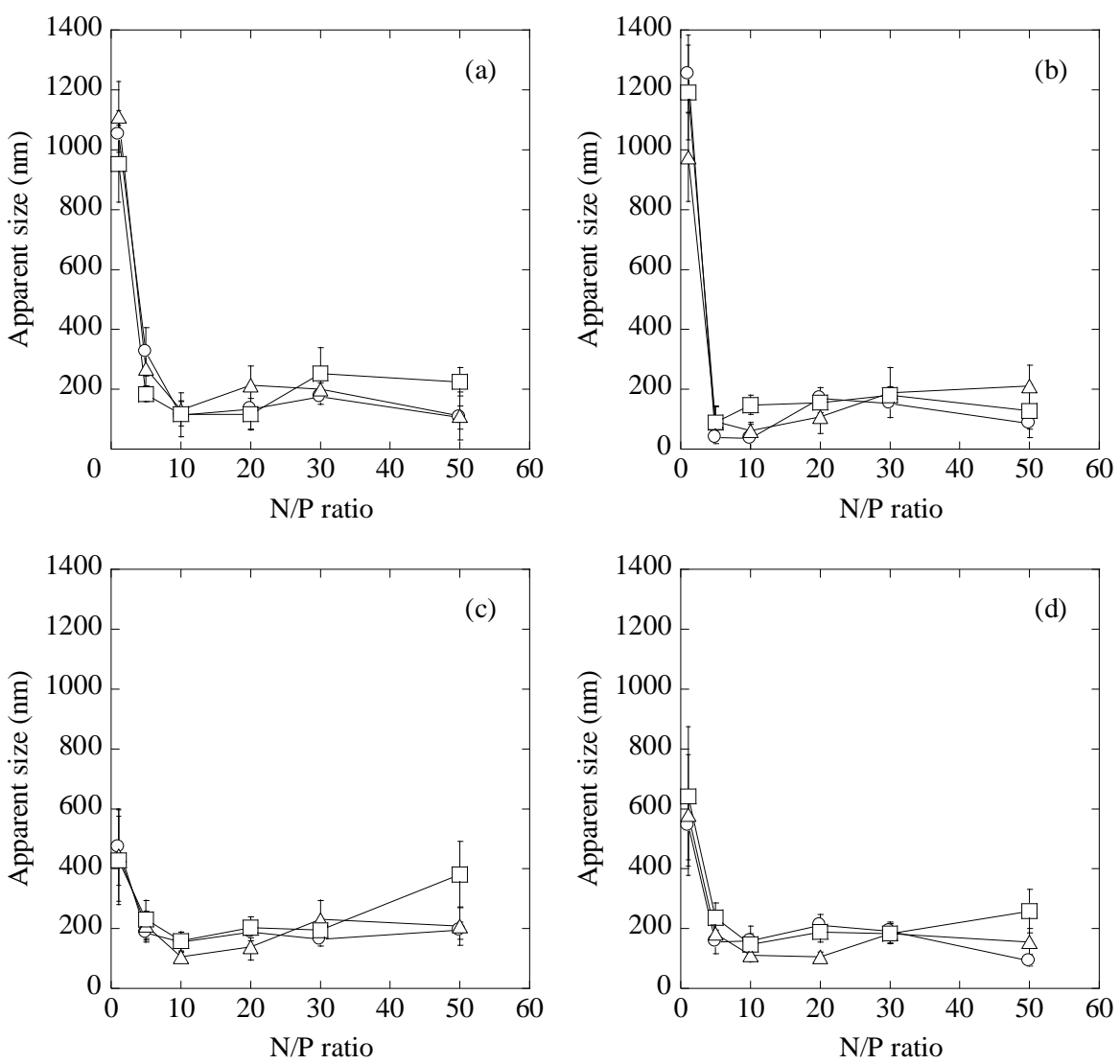

Figure $1(\mathrm{~A})$
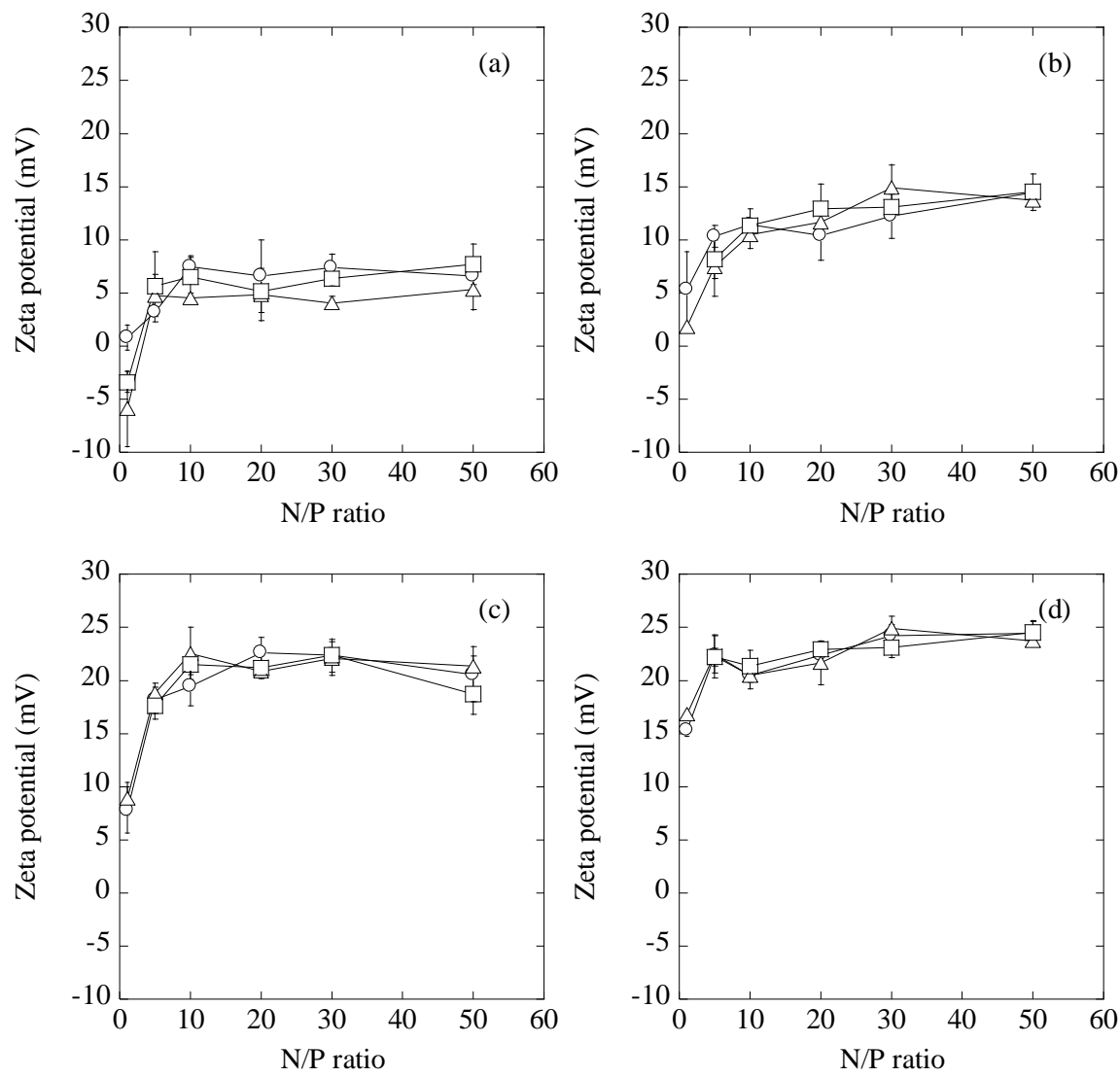

Figure 1 (B) 


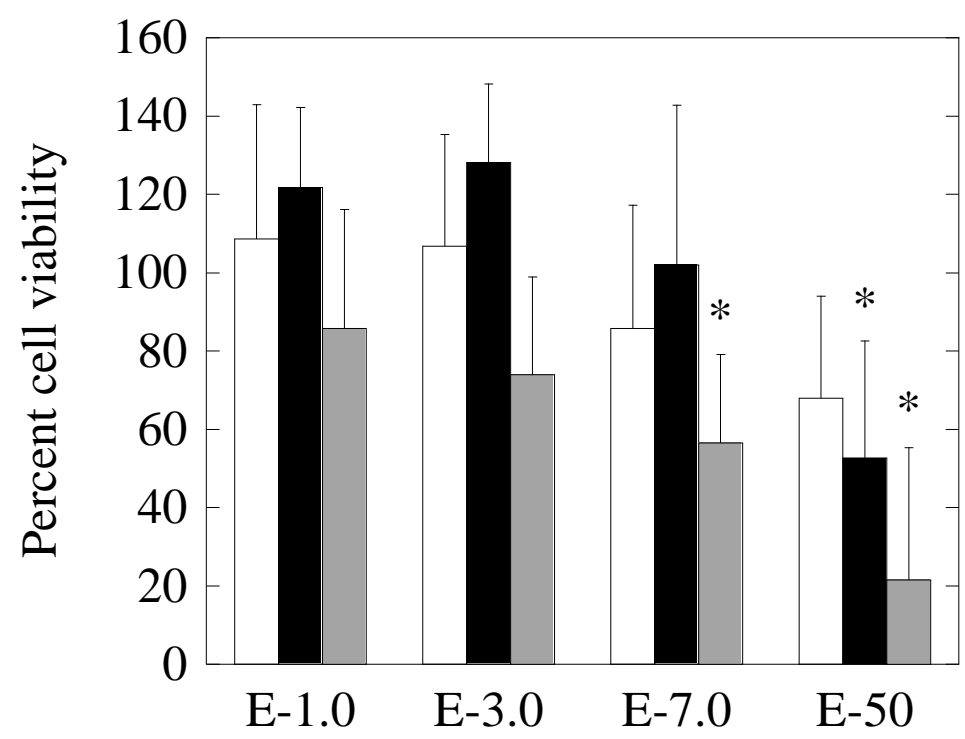

Figure 2 

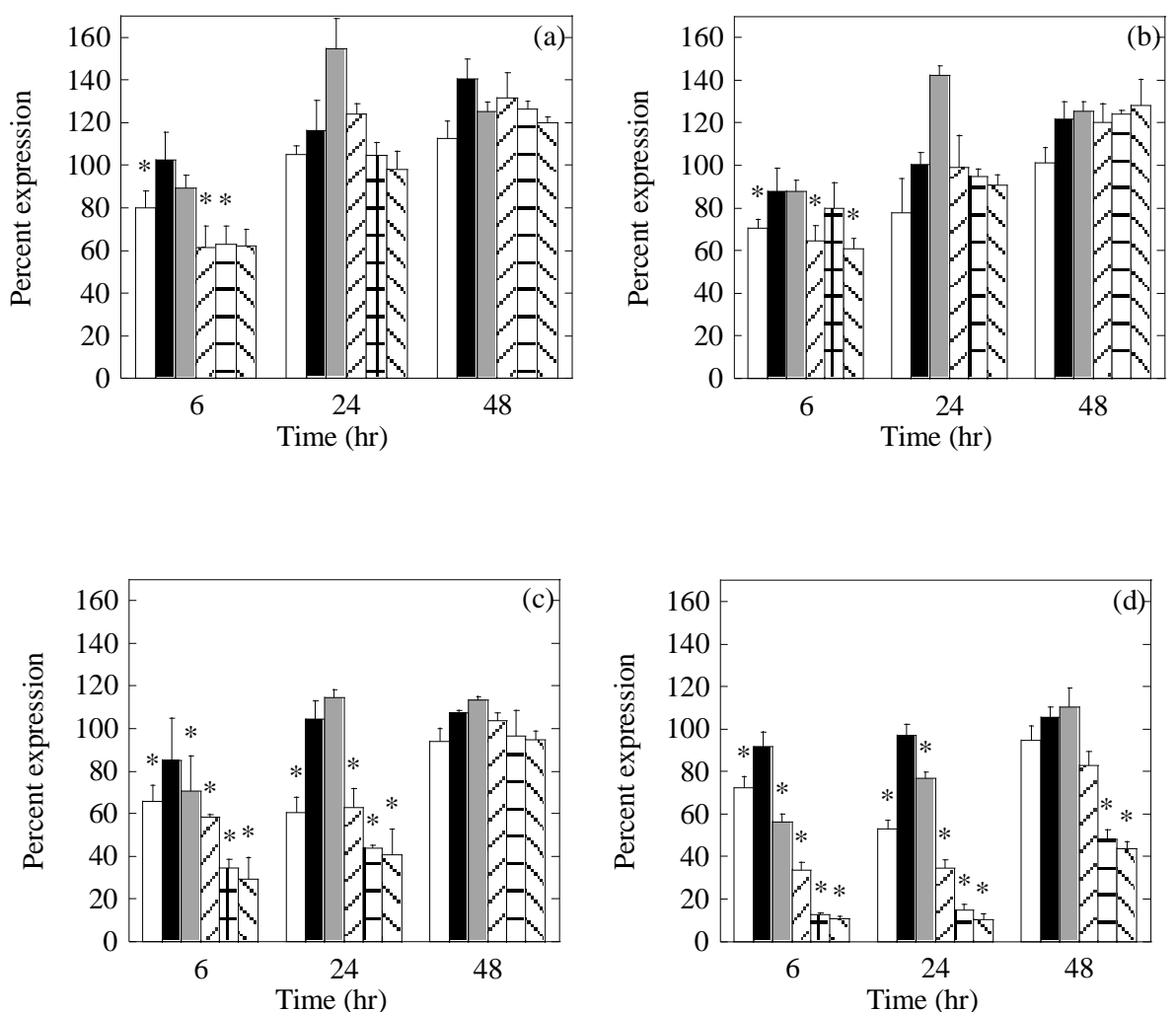

Figure 3 

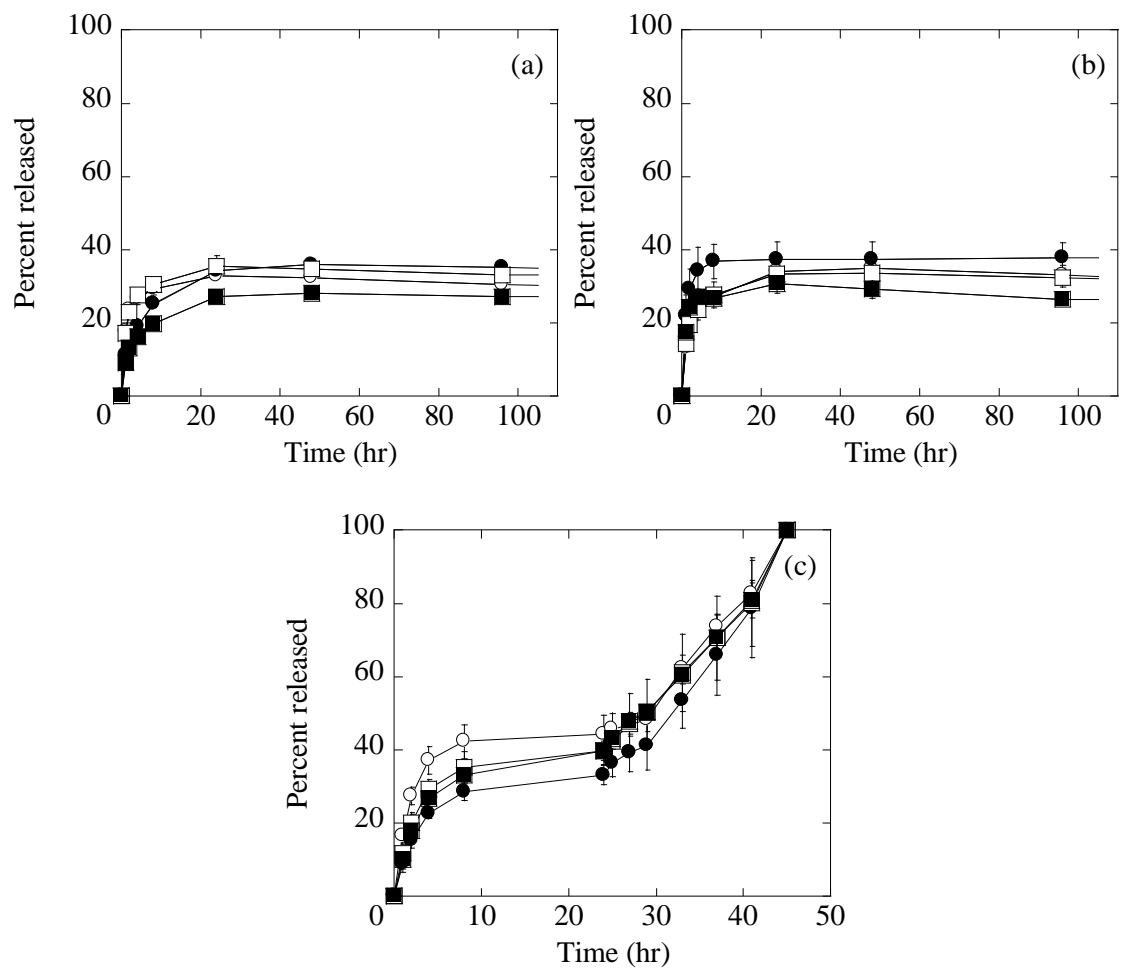

Figure 4 


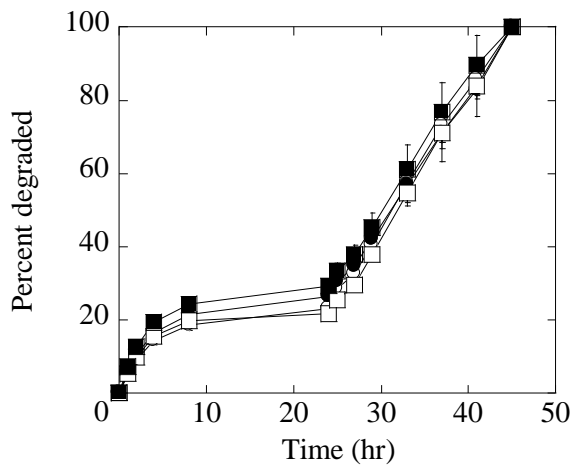

Figure 5 

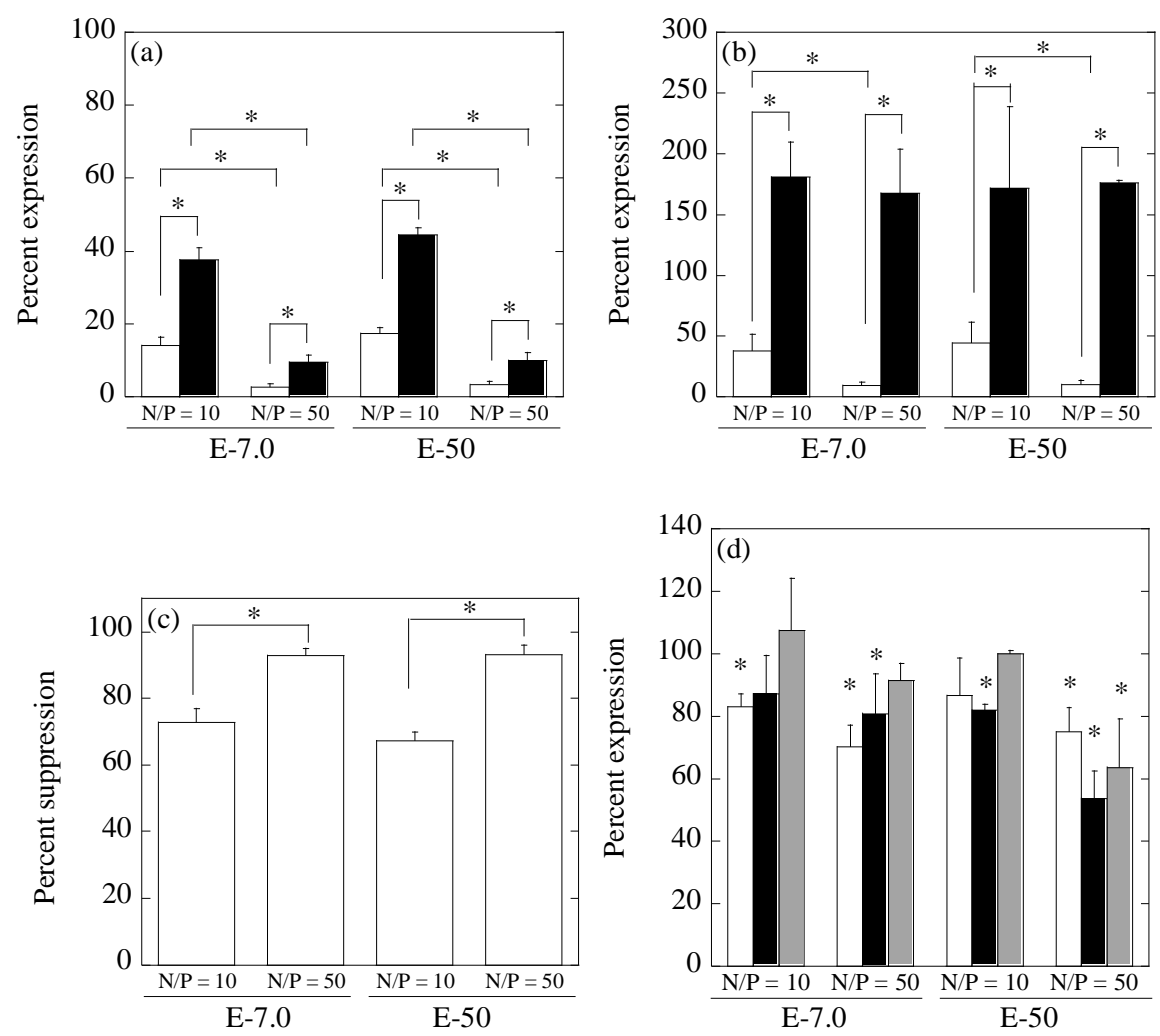

Figure 6 


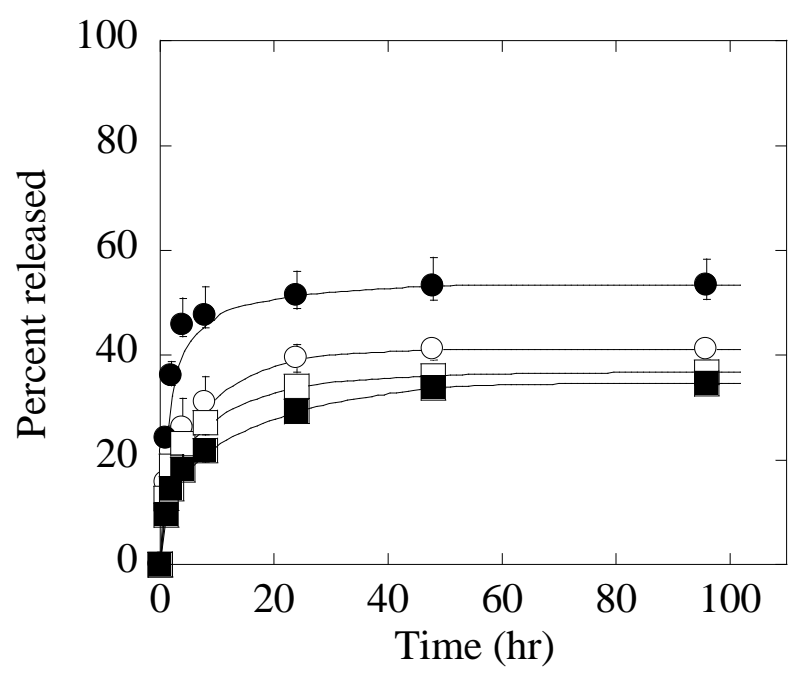

Supplementary Figure 1. In vitro release profiles of Luc siRNA from gelatin hydrogels incorporating complexes of Luc siRNA and CG. The complexes were prepared for siRNA with E-1.0 (○), E-3.0 (O), E-7.0 ( $\square)$, and E-50 CG $(\boldsymbol{\square})$. The N/P ratio is 50. 